Cinémas

Revue d'études cinématographiques

Journal of Film Studies

\title{
Tactiques scripturaires dans les cinémas d'Afrique noire
}

\section{De B’béri Boulou Ebanda}

Volume 11, numéro 1, automne 2000

Écritures dans les cinémas d’Afrique noire

URI : https://id.erudit.org/iderudit/024831ar

DOI : https://doi.org/10.7202/024831ar

Aller au sommaire du numéro

Éditeur(s)

Cinémas

ISSN

1181-6945 (imprimé)

1705-6500 (numérique)

Découvrir la revue

Citer cet article

Boulou Ebanda, D. B. (2000). Tactiques scripturaires dans les cinémas d'Afrique noire. Cinémas, 11(1), 11-30. https://doi.org/10.7202/024831ar

\section{Résumé de l'article}

Notre réflexion sur le cinéma d'Afrique noire trouve son point de départ dans les concepts de stratégie et de tactique, que nous empruntons à Michel de Certeau. Notre hypothèse est que les cinémas d'Afrique noire s'infiltrent dans les structures stratégiques des cinémas dominants, créant ainsi de nouvelles voies d'analyse. Un survol historique des images et discours sur l'Afrique montrera comment s'opèrent la réappropriation et la rappropriation de l'image du Noir par le Noir. La description de deux séquences de films montrera comment la rappropriation s'effectue par des détournements fortement politisés. 


\title{
Tactiques scripturaires dans les cinémas d'Afrique noire
}

\section{De B’béri Boulou Ebanda}

\begin{abstract}
RÉSUME
Notre réflexion sur le cinéma d'Afrique noire trouve son point de départ dans les concepts de stratégie et de tactique, que nous empruntons à Michel de Certeau. Notre hypothèse est que les cinémas d'Afrique noire s'infiltrent dans les structures stratégiques des cinémas dominants, créant ainsi de nouvelles voies d'analyse. Un survol historique des images et discours sur l'Afrique montrera comment s'opèrent la réappropriation et la rappropriation de l'image du Noir par le Noir. La description de deux séquences de films montrera comment la rappropriation s'effectue par des détournements fortement politisés.
\end{abstract}

\begin{abstract}
Our research on Black African cinema takes as its starting point the concepts of strategy and tactics, which we borrowed from Michel de Certeau. Our hypothesis is that Black African cinemas infiltrate the strategic structures of dominant cinemas, thereby opening up new paths for analysis. A historical overview of images and discourses about Africa will show how the reappropriation and rappropriation of the image of Blacks by Blacks themselves operate. The description of two film sequences will then show how reappropriation functions through strongly politicized diversions.
\end{abstract}


Rappel historique : l'Afrique, du cinématographe au cinéma

L'oubli ou la méconnaissance du sujet historique a pour conséquence de réduire à une universalité vague et abstraite la particularité qu'on revendique.

F. Eboussi Boulaga

Il est important d'inscrire dans son contexte historique le processus de réappropriation et de rappropriation du cinéma par les Africains. À cet égard, deux périodes sont marquantes. La première, celle du cinématographe, est caractérisée par un cinéma zoologique, c'est-à-dire qui s'attache à produire des images spectactulaires. La seconde période, celle du cinéma d'art, est aussi celle de la réaction des Africains aux images jusque-là proposées de leur société. Explorant l'espace de l'art, ils s'engageront dans l'aventure cinématographique avec la conviction que le média leur permet de dire, eux-mêmes, ce qu'ils sont véritablement.

\section{- L'AFRIQUE ANTHROPOLOGIQUE DU CINÉMATOGRAPHE}

Les histoires du cinéma ne le mentionnent guère, mais l'Afrique fut l'un des espaces les plus filmés, après la naissance du cinématographe. Des "documentaires", des fictions même, y furent tournés par des pionniers du cinéma parmi lesquels certains sont notoires. Ainsi, vers 1905, Méliès signait Le Marché de Dakar et Cake-walk de nègres du nouveau cirque. Guido Convents (1989, p. 60) souligne aussi qu'entre 1899 et 1901 , dans le sud du continent africain où sévissait la guerre des Boers, abondaient les opérateurs occidentaux avides de produire des images spectaculaires destinées aux fêtes foraines. Ce cinéma des premiers temps fait en Afrique développe jusqu'au moment du parlant - vers 1926, 1927 - ce goût du spectaculaire dont l'Afrique fait les frais. Il s'agit d'« un cinéma de pacotille, où primitifs et cannibales sont jetés en pâture à la curiosité et à l'effroi des publics occidentaux" (Debrix, p. 7). 
Ainsi l'Afrique était-elle bien présente en Occident au début $\mathrm{du} \mathrm{XX}^{\mathrm{c}}$ siècle, mais sous la forme du spectaculaire exotique. En témoigne l'article intitulé "La maladie du sommeil» que l'on trouve dans la rubrique scientifique du Pathé Journal de décembre 1912, article signé par un certain docteur J. C. :

Notre bande cinématographique qui paraît en cinquième page est consacrée à la maladie du sommeil. On y voit un pauvre nègre, d'une maigreur squelettique, atteint de cette infection. Il présente sa face hébétée à l'objectif photographique du courageux opérateur qui osa traverser les régions maudires où règne la mouche tsé-tsé, la redoutable tsé-tsé: glossina palpalis, comme nos savants la nomment. Le film se déroulant, la projection nous donne ensuite la vision infernale que présente, à un grossissement de trente mille diamètres, l'infime partie d'une goutte de sang d'un malade victime de la mouche [...].

Après avoir associé par métaphore les microbes et les lilliputiens d'une part, les globules blancs et rouges d'autre part, l'auteur précise que les vigilants globules blancs sont les protecteurs du sang des nègres ainsi infectés. Puis il poursuit :

Beaucoup d'entre nous, dans leurs jeunes années, se sont, sans doute, enthousiasmés aux récits des voyages à travers l'Afrique, à la grande randonnée de Livingstone, aux aventures des chasseurs de girafes ou des jeunes Boers contées par Mayne Reid. Ils ont donc entendu parler cle la mouche tsé-tsé. Ils savent combien les colons, les explorateurs de l'Afrique la craignaient, non pas pour eux, il est vrai, mais pour leurs chevaux, leurs troupeaux qui étaient infailliblement décimés quand on passait dans les pays habités par cet insecte. [...] Ma causerie s'est assez prolongée et ces questions, heureusement, ri'intéressent que peu de nos lecteurs, habitants de la France, où le sommeil est non pas une maladie, mais une très douce chose.

On le constate, le spectaculaire africain est l'occasion d'affirmer la supériorité occidentale. C'est d'ailleurs cet exotisme africain que mirent en scène les expositions coloniales. En France, 
celle de 1931 fut l'occasion pour des millions de visiteurs de partager [ $[\ldots]$ une même fascination des mystères des colonies et une même fierté de la marque de l'homme blanc sur tant de mers et de continents" (Hodeir et Pierre, 1991, p. 138). Dans ce contexte, c'est haut et fort que s'affirme la visée colonialiste. Le 2 juillet 1931, Paul Reynaud, alors ministre des Colonies, déclarait aux journalistes que " [la] colonisation est un phénomène qui s'impose, car il est dans la nature des choses que les peuples arrivés à un niveau supérieur d'évolution se penchent vers ceux qui sont à un niveau inférieur pour les élever jusqu'à eux" (Hodeir et Pierre, 1991, p. 139). Louis XIV et Louis XV ne disaient pas autre chose dans leurs codes du Noir.

C'est dire que les images récoltées par les opérateurs participèrent du colonialisme. À cet égard, un article intitulé «Le cinématographe à la conquête du monde " paru en 1909 dans CinéJournal est significatif. En effet, l'auteur élève l'opérateur au rang de héros épique:

Nous verrons, dans un laps de temps plus ou moins rapproché [...] le marché du cinématographe envahi par d'innombrables films exotiques [...]. On a déjà eu un avant-goût par l'édition de certaines bandes reproduisant des scènes sous les tropiques, des paysages et des traits de mœurs enregistrés au cœur de l'Afrique [...]. Les expéditions cinématographiques [...] sont de véritables voyages d'exploration entourés de tous les dangers. [...] Il faut au "Chasseur de Films" dans la brousse ou le désert, dans les gorges sauvages et abruptes ou dans la forêt vierge, plus que du courage et du sang froid. [...] L'explorateur ordinaire doit s'attendre à entrer en lutte avec les intempéries, contre les maladies, avec les fauves, avec les obstacles naturels dont sa route est hérissée. Mais s'il peut les combattre, il peut, dans une certaine mesure, les éviter, les circonvenir. Il en est autrement de l'explorateur doublé d'un opérateur cinématographique ou si l'on préfere l'opérateur doublé d'un explorateur, ce qui est plus juste au fond. Celui-ci n'a pas le choix des moyens. Au lieu de fuir le danger, il lui faut le souhaiter, le désirer, aller au-devant de lui, le provoquer même, et s'exposer à tous les risques et à tous les dangers, dans le seul but de rapporter une ample moisson de films, utilement impressionnés. [...] Et 
ce ne sont pas toujours les fauves que l'on chasse et que l'on tue, que l'on immole sur l'autel du dieu Cinéma comme un sacrifice agréable à l'écran qui sont le plus à redouter; il y a aussi les peuplades sauvages, guerrières et farouches, qui guettent l'opérateur-explorateur sur son passage et lui font, de concert avec les fièvres du sommeil ou les insectes venimeux, une guerre à outrance $[. .$.$] .$

Les images ramenées d'Afrique eurent un tel succès qu'en 1929, un article de Cinémonde, signé M. G., avait pour titre «Le noir est à la mode». L'auteur de cet article considère l'Afrique comme un "[...] réservoir inouï de lyrisme, de rêve [...]", comme un paradis retrouvé propre à inspirer les plus grandes créations. Ici, c'est de conquête artistique qu'il s'agit.

Mais l'Afrique, qu'elle apparaisse comme une terre idyllique ou barbare, qu'elle constitue une occasion d'évasion ou de conquête, et peu importe le type de conquête qu'elle inspire, reste une Afrique construite et pensée par les Occidentaux. Et en cela, les images ramenées du continent africain n'ont fait que s'inscrire dans une longue tradition. Qu'elles aient été tournées par des prêtres, des anthropologues, des ethnologues, des colonialistes ou opérateurs de tout acabit, c'est toujours et partout, au mieux, l'exotisme et le regard de surplomb qui dominent, tant au tournage qu'à la réception. Et la critique cinématographique ne fait pas exception.

Cette représentation de l'Afrique est d'une importance capitale pour les Africains. En effet, elle est un point de départ incontournable, car c'est à partir d'elle qu'ils mettront en action un mode de réception hautement actif. Autrement dit, en plus de lire dans les critiques l'intérêt que le public occidental porte aux images des sauvages d'Afrique, les Africains liront pour leur propre compte les modes opératoires des images sur lesquelles portent ces discours. C'est après l'examen du contenu de ces discours sur l'homme noir, discours faits de langage et d'images, que la prise de conscience des Africains prendra la forme d'une attaque. Cette attaque sera une réponse pour contrecarrer ces regards portés sur l'Afrique et le Noir, occasion pour les Africains de dire eux-mêmes ce qu'ils sont. Elle consistera en une écriture 
de réappropriation des images africaines. Du coup, elle constituera une entreprise de rappropriation - heureux belgicisme, qui permet de désigner la nécessité pressante de rendre propre ce qui a été sali.

\section{- DE LA NÉGRITUDE AU CINÉMA, UNE RÉACTION POLITIQUE}

On vient de voir que les premières images des Africains ont été produites par d'autres qu'eux. La réponse africaine, «l'autre voie" comme le disait Ousmane Sembène, voulait donc contrecarrer ces premiers regards. Mais la revendication d'un autre regard n'est pas née du cinéma. Elle émerge avec les premiers intellectuels noirs. Maîtrisant la rhétorique du verbe occidental, ces écrivains ont su se faire entendre de ceux qui les regardaient. Dans un premier temps, leurs productions n'étaient pas destinées aux Africains - vu le nombre élevé d'analphabètes et d'illettrés -, mais bien au monde extérieur. Il s'agissait pour eux de dire ce qu'ils étaient véritablement.

Retenons 1921 comme une année importante dans ce processus d'affirmation. C'est l'année où pour la première fois le Goncourt est décerné à un Noir. Il s'agit de l'Haïtien René Maran (1887-1960), qui reçoit le prix pour son livre Batouala, véritable roman nègre. Cette reconnaissance de la littérature noire en Occident a certainement beaucoup à voir avec la formulation et le développement du concept de "négritude», puis avec l'essor des cinémas d'Afrique noire. Car elle porte un fait au jour : la maîtrise des langues coloniales par les indigènes mène à la contre-pénétration de ces indigènes dans les espaces auparavant réservés aux colons. La maitrise des langues et techniques des colons permet aux Noirs de dynamiter, de l'intérieur même des espaces coloniaux, les discours qui y sont produits contre eux. Comme le mentionne Lilian Kesteloot (1968, p. 6), "[...] avec fermeté, Maran remettait en question la mission civilisatrice et coloniale de l'Occident". C'est de cela que se nourrira la pensée des écrivains de la Négritude, axée sur la revendication d'un autre regard, sur une implication qui demande à chaque Homme Noir de dire ce que personne d'autre ne peut dire sur sa civilisation. 
C'est dire que la maîtrise du langage ne fait pas que mener à la contre-pénétration des colonisés dans les espaces auparavant exclusifs aux colons. Car ce qu'elle indique, c'est une réception active dont elle n'est que l'un des degrés les plus bas, le plus élevé se manifestant par une réappropriation du langage qui entraîne des activités de détournement. En d'autres termes, les langues coloniales, d'abord imposées, se trouvent ensuite affectées à la définition d'une image propre, ce que nous appelons ici une rappropriation.

Cette thèse invalide celle de Stuart Hall (1980), selon laquelle l'intégration du discours dominant par une instance de réception impliquerait qu'elle opère avec les codes de référence contenus dans ce discours. Les Africains participant à l'étude et à la maîtrise du discours dominant ne pourront pas être qualifiés de "public participant», car l'action finale de cette participation est en réalité une opposition. Leur pénétration forcée dans les espaces coloniaux est plutôt une "séduction" qui leur permet de maîtriser les techniques langagières des colonisateurs. Ensuite suivra un mouvement de décolonisation, une "coerséduction" (Ravault, 1986). Et c'est à ce moment-là que les instruments qui contrôlaient les images des Africains seront détournés pour créer et imposer un regard propre. La création de la FEPACI (Fédération panafricaine de cinéastes), par exemple, relève d'un tel détournement. Les cinéastes se sont dotés de cette institution pour lutter contre l'impérialisme et le néocolonialisme. Et s'il est écrit dans la charte de l'organisme que "[l]'image stéréotypée du créateur solitaire et marginal, répandue dans la société capitaliste occidentale, doit être rejetée par le cinéaste africain [...]" (Alger, 1975), c'est que ses membres craignent que leurs propres créations ne servent aux discours qu'ils veulent rejeter.

\section{- AU-DELÀ DU POLITIQUE DANS LA CRÉATION, LA CONTRE-RHÉ IORIQUE}

La charte de la FEPACI, si elle semble limiter la liberté de création, présente un aspect politique qui permet de comprendre l'esprit dans lequel les cinéastes circonscriront leur écriture. Pour ce faire, il faut tenir compte au moins de deux facteurs. 
Le premier, c'est la domination des compagnies étrangères de distribution sur les marchés locaux. En effet, les pays africains qui décidaient de la distribution à l'intérieur de leurs propres frontières étaient sanctionnés. Ce trust étranger imposait aussi un contenu cinématographique précis: les films dénonçant les maux du colonialisme et les regards anthropologiques des Occidentaux étaient automatiquement censurés. C'est justement la tension régnant entre les cinéastes africains et le représentant du ministère de la Coopération française que F. Boughedir filme, dans Caméra d'Afrique (1982). Le principal bailleur de fonds du cinéma francophone, la France, ne voulait plus financer un cinéma continuellement contestataire de son passé colonialiste. L'autre facteur, c'est le fait que le cinéma soit considéré par les gouvernements africains et les hommes de pouvoir traditionalistes comme un instrument de persuasion. Par conséquent, les artistes auront aussi à se battre contre ces instances internes. Ainsi, tout film qui relate la mauvaise gestion de l'état ou qui critique les formes de pouvoir traditionnel a toutes les chances de finir ses jours au placard.

C'est à cette censure "en règle" que s'oppose la FEPACI. Toutefois, il faut mentionner que la double censure a eu un effet bénéfique sur l'écriture de certains cinéastes. En effet, il arrive qu'une articulation langagière profondément rhétorique voile à la fois les thématiques et contenus de films contestés par les bailleurs de fonds occidentaux et ceux décriés par les élites internes. On trouve un exemple de cette rhétorique dans la subtilité du langage d'un film comme Wend Kuuni (1982) de Gaston Kaboré. On n'y maudit plus ouvertement le colon, mais on dit: "C'était avant l'arrivée des Blancs '."

Ce bref développement historique nous conduit à examiner pratiquement les modes opératoires de l'écriture dans les cinémas d'Afrique noire, et nous allons le faire en impliquant les concepts de "stratégie" et de "tactique" dans une analyse comparative de deux séquences de films.

\section{Stratégie scripturaire des cinémas dominants}

C'est chez Michel de Certeau (1990, p. XLVI) que l'étude qui suit prend son point de départ: 
J'appelle "stratégie" le calcul des rapports de forces qui devient possible à partir du moment où un sujet de vouloir et de pouvoir est isolable d'un "environnement». [La stratégie] postule un lieu susceptible d'être circonscrit comme propre et donc de servir de base à une gestion de ses relations avec une extériorité distincte. La rationalité politique, économique ou scientifique s'est construite sur ce modèle stratégique.

En ce qui concerne le cinéma, nous assimilons l'écriture stratégique à l'organisation discursive basée sur le logos poétique, lequel illustre la transparence d'un discours rationnel et circonscrit l'événement comme un propre. Bref, l'écriture stratégique serait de l'ordre de la représentation. Et nous soutenons que la stratégie serait le propre d'une gestion organisée des opérations de production dans les cinémas dominants. Cette gestion gomme toutefois l'espace propre de l'événement et articule son écriture sur un langage institutionnalisé qui laisse l'actualisation de toute la production - même des non-dits - à l'instance de réception. Ce langage ancré dans les cinémas dominants se précise par exemple avec l'utilisation des champs-contrechamps, des ellipses, ou de toute autre figure issue des codes et pratiques instrumentaires et institutionnalisés qui légitiment un discours devenu rationnel. Afin de voir comment cette stratégie scripturaire fonctionne pratiquement, décrivons une séquence de L'Enfant noir (1995), une adaptation cinématographique du roman de Camara Laye par un Français, Laurent Chevalier.

L'Enfant noir, c'est l'histoire d'un enfant qui quitte son village pour aller habiter chez son oncle, à la ville, histoire de poursuivre des études. Avant le départ de l'enfant, son père va consulter un marabout. Ce sont les sept plans constituant cette séquence chez le marabout que nous voulons analyser: 1 . Son off sur un plan de paysage (un arbre). La voix off de l'enfant raconte que rien ne peut se faire dans son village sans consulter le vieux Massini (le marabout). 2. Gros plan du visage du marabout qui regarde de part et d'autre. Il balance le bras de gauche à droite, en l'air, mais on ne sait pas ce qu'il regarde et on voit à peine ce qu'il tient dans les mains. 3. Gros plan du visage du père de l'enfant chevauché par la voix off du marabout disant : "Tu 
sacrifieras une chèvre et un coq rouge..." 4. Plan rapproché (poitrine) du marabout, il joue de la kora et chante. En voix off, l'enfant explique qui est le vieux marabout. 5. Gros plan du visage du marabout. Il regarde à terre, secoue des objets qu'on ne voit toujours pas. Il lève la tête et regarde dans l'espace de la pièce. On ne voit toujours pas cet espace. 6. Plan rapproché (poitrine) du père, il regarde le marabout. 7. Plan rapproché du marabout, il rejoue de la kora.

Ces sept plans composent toute la séquence. Leur échelle et leur durée (deux à quatre secondes) influent sur l'espace. Nous le savons, une des principales caractéristiques du gros plan est d'éliminer l'espace. Ce choix de cadrage n'est pas anodin, qui met l'accent sur l'action de la représentation aux dépens de cette scène qui, si elle voulait traduire ou reproduire l'expérience (la consultation d'un marabout), permettrait tout au moins de contempler l'espace et les objets cultuels au lieu de les gommer. Ici, l'espace, dans ce qu'il a de sacré, est complètement éliminé. C'est la séquence classique de la communion dans une église. Gommant l'espace sacré du lieu, l'environnement mystique, «le sujet productant" - un sujet de vouloir et de pouvoir, d'après De Certeau - décide de montrer en gros plan les visages des protagonistes, prêtre et communiant.

Umberto Eco (1985, p. 21) affirme « [qu'il] n'est pas d'énoncé qui, pour être sémantiquement actualisé dans toutes ses possibilités de signification, ne requière un co-texte». Ici, le co-texte désigne une instance de réception qui, avec son propre bagage référentiel, actualise toute la signification d'une production. Mais à quel bagage référentiel cette instance de réception doit-elle faire appel? Dans l'introduction de son livre sur l'orientalisme, Edward Saïd fait un constat important $(1978$, p. 1) :

On a visit to Beirut during the terrible civil war of 1975-1976 a French journalist wrote regretfully of the gutted downtown area that " it had once seemed to belong to [...] the Orient of Chateaubriand and Nerval". He was right about the place, of course, especially so far as a European was concerned. The Orierit was almost a European invention, and had been since antiquity a place of romance, exotic beings, haunting memories 
and landscapes, remarkable experiences. Now it was disappearing [...]. Perhaps it seemed irrelevant that Orientals themselves had something at stake in the process [...]; the main thing for an European visitor was a European representation of the Orient and its contemporary fate, both which had privileged communal significance for the journalist and his French readers.

Pourra-t-on, après ce constat de Saïd, soutenir que la lecture qu'un spectateur français fera de la scène de L'Enfant noir sera issue des images propres de cette pratique de consultation des voies mystiques? Et en supposant que cette représentation permette à l'instance de réception, comme l'a soutenu Umberto Eco, d'actualiser son encyclopédie, à partir de quelle sphère de connaissances cette encyclopédie se trouvera-t-elle actualisée, celles issues d'un discours préalablement ancré dans sa culture ou celles qui lui sont offertes par la production? En d'autres termes, le spectateur français sera-t-il capable d'actualiser la spatiotemporalité et la pratique de ce rituel de consultation d'un marabout en faisant impasse sur le discours français préexistant et qui créa cette consultation comme un objet de représentation?

Dans cette scène de L'Enfant noir, un langage cinématographique non stratégique serait tout autre. Nous sommes chez un marabout, dans une pièce qui préfigure une espèce de saint des saints. Le narrateur, la voix off de l'enfant, nous le dit d'ailleurs explicitement: "Dans notre village, personne n'entreprend jamais quelque chose sans consulter le vieux Massini! "Cet énoncé marque le statut sacral du lieu, le statut social du marabout et celui de l'acte de consultation des voies mystiques. Contrairement à un traitement spatio-temporel qui n'offre aucune donnée pouvant nous permettre de faire cette lecture, nous allons donc l'explorer, "voir" les objets qui caractérisent dans notre "conscience affective " le saint des saints d'un marabout. Mais le point de vue de Chevalier, axé sur la stratégie institutionnalisée des cinémas dominants, écarte cette potentialité. Son langage cinématographique - gros plans, champs-contrechamps, montage rapide, etc. - vide la scène de toute sa signifiance intrinsèque.

C'est l'éloge de la pseudo-ubiquité de la représentation. Une articulation langagière qui légitime un discours instrumental à 
travers lequel la "vitesse-action" extériorise le pouvoir de représentation d'un sujet productant dont la vision domine le véritable rythme de l'événement. Une représentation qui favorise le dramatique (action) aux dépens de l'étant identitaire (acte d'être) de l'action. C'est ainsi que le cadre reste serré, car jouant avec une écriture institutionnalisée. Parallèlement, cette stratégie répond aussi aux questions théoriques des narratologues et sémiologues du cinéma qui demandent: qui raconte? C'est le "sujet», l'instance de pouvoir, le méga-narrateur, l'énonciateur omniscient, le grand imagier, etc.

Les visages du père de l'enfant et celui du marabout ressortent gros plan après gros plan. Ce sont les yeux, les dents, les rides qui sont au centre de l'image, alors que ce qui semble refléter l'expérience de ce rituel est dans l'environnement gommé. Toute la gestuelle ritualisée du marabout qui tourne son regard de part et d'autre vers des points précis de l'espace, toute la gestuelle des mains tenant des objets cultuels et mythiques, celle des bras fendant l'air, celle des regards implorant, invoquant, ne font pas partie du langage cinématographique de Chevalier, et ainsi, elles ne sont pas données à voir.

Pour ceux et celles qui connaissent le roman de Camara Laye, il sera incontestable que son adaptation par ce cinéaste français marque une écriture cinématographique pauvre d'acceptions scripturaires propres à l'Afrique. Bien que notre analyse ne porte pas sur l'adaptation, nous avançons qu'en favorisant son point de vue comme celui d'un narrateur omniscient, légitimé par une stratégie scripturaire institutionnalisée, Chevalier manque totalement ce dont le livre traite: la pratique orale, le pouvoir de la parole, la séparation pénible d'un enfant d'avec sa mère..., des données qui ont des liens profonds avec la tradition et qui étaient au centre de l'écriture de Laye.

Ce serait par ailleurs une excuse de considérer la méconnaissance des traditions africaines comme une cause déterminante de cette écriture. Autrement dit, nous ne pensons pas, comme le soutiendraient les affamés de la représentation, que Chevalier utilise ici un langage représentant une chose qui aurait pu par ailleurs être présentée de multiples manières. La question nous semble ailleurs. Car d'une part, les limites de cette écriture ex- 
posent celles des modes opératoires stratégiques qui la structurent. D'autre part, cette écriture sous-tend un langage préalablement contarniné par une représentation issue d'un vieux discours, d'un habitus langagier français qui a lui-même déjà réifié les espaces cultuels du "maraboutisme" en Afrique. Chevalier ne pouvait donc pas localiser le sens de cette séquence autrement. Il ne pouvait même pas représenter cette consultation autrement, sinon qu'avec les limites référentielles de son encyclopédie.

Ainsi, même si cette écriture apparaît "ouverte" au point de vue de la lecture - en effet, elle est pleine de non-dits (ellipses temporelles et spatiales, etc.) —, elle demeure une écriture qui ne permet pas aux spectateurs de lui donner une vie intrinsèque. Et c'est ici un paradoxe épistémologique qui échappe encore aux théoriciens de la réception. En reprenant les termes de De Certeau, on se rend compte que "la stratégie postule un lieu susceptible d'être circonscrit comme propre", comme un propre, mais ce lieu n'est pas le propre, il n'atteint pas au propre de l'événement. Le destinataire contribuera donc à remplir les ellipses d'images de "sa. propre " encyclopédie, de "ses propres" impacts émotifs et de "ses propres" suggestions extralinguistiques. Mais tout cela est déjà contaminé par un discours préexistant qui a construit les schémas et images de cette représentation. Et il en va de même pour le "sujet productant".

Enfin, dans cette écriture stratégique, nous retrouvons toujours la prédominance des rapports communicatifs érigés par un logos poétique ${ }^{4}$. Ainsi, l'obligation de communication vient favoriser une transparence extérieure à l'objet audiovisuel, mais qui légitime l'écriture de l'énonciateur. La communication devient elle-même saisissable en tant que communication à partir de sa réception. Ici, le "sujet" organise le destinataire en privilégiant des accès de sens, des formes particulières et des plages langagières. Toute la structure de ce discours dans les arts, cette grammaire rationnelle et institutionnalisée, c'est ce que De Certeau a appelé "stratégie ». Comme dans cette séquence de L'Enfant noir, le sujet de pouvoir dirige, monte et démonte, croise et entrecroise les données et impose son cadrage, son point de vue, tout en construisant parallèlement une communication avec la 
réception. Mais en dehors de cette stratégie scripturaire, quelles sont les autres possibilités d'écriture?

\section{Tactique de détournement des cinémas d'Afrique noire}

Afin de voir comment le concept de "tactique» devient le propre des cinémas d'Afrique noire, nous allons maintenant analyser une autre séquence d'un film qui traite de l'acte de consultation chez un marabout. Le Gardien des forces (1990) de la Togolaise Anne-Laure Folly a obtenu le prix Images des femmes au festival Vues d'Afrique de Montréal en 1992.

La séquence que nous avons choisie montre aussi l'acte de consultation des voies mystiques et contient sept plans comme celle de L'Enfant noir: 1. Voix off du marabout sur un travelling arrière en plan très large sur une cour d'habitation. La voix off dit: "Chez nous les morts ne sont pas morts..." 2. Plan d'ensemble du lieu de consultation. Le marabout est à la droite de l'écran, son aide est à sa gauche et il y a un fétiche au centre de l'image (l'aide du marabout parle et tape sur le sol). 3. Gros plan d'un fétiche accroché sur le mur. 4. Travelling et zoom arrière en plan très large de la salle. On voit le marabout et une autre personne (une fernme) qui écoutent les incantations de l'aide-marabout (voix off de la narratrice qui explique aussi ce qu'est un fétiche). 5. Plan large de l'aide-marabout faisant toujours des incantations sur le fétiche. L'aide est toujours accroupi et occupe la même position à gauche du cadrage. Le fétiche est toujours au centre du cadre. 6. Gros plan sur le fétiche (voix off de la narratrice qui continue d'expliquer ce que c'est qu'un fétiche). 7. Gros plan suivi d'un zoom in en très gros plan sur un autre fétiche dans la même salle.

Il ne s'agit pas seulement de montrer que cette séquence comporte moins de gros plans que celle de L'Enfant noir, quoique cela joue un rôle important lié à sa vitesse-lenteur. Contrairement au gros plan, le plan large, en soi, impose un certain temps, long, étant donné qu'il met en image plusieurs éléments qu'il faut ensuite avoir le temps d'absorber, de lire. Pour que le destinataire fasse toute la lecture nécessaire et intrinsèque aux images et à l'espace qui lui sont proposés, les choix scripturaires de cette séquence offrent un temps pour la contemplation. Il faut vivre avec... 
Dans les sphères discursives de la communication dominante, d'aucuns analyseront cette vitesse-lenteur comme un manque de rythme ou la lieront à une question d'économie de la production. Mais comme nous le dit Andrée Devanture, monteuse française bien connue des cinéastes africains, "[...] ce principe de film lent laisse au spectateur la liberté de voir ce qu'il a envie de voir, de s'impliquer, de découvrir. Son imaginaire peut fonctionner" (Barlet, 1996, p. 188). Ce constat est fort important car, pour les spectateurs et artistes africains, tout est en grande partie un facteur de "mémoire", d'un imaginaire qui mène à faire revivre, à actualiser à travers la "conscience collective" une "affectivité " qui permettra à chaque Africain d'occulter "la mort de sa mémoire». Et nous ne l'oublierons pas, comme pour les écrivains de la Négritude, il s'agit aussi pour les cinéastes de dire ce que personne d'autre ne peut dire à leur place.

La construction scripturaire de cette séquence offre donc une place importante à la contemplation des espaces et des objets cultuels dans cet espace. Ce traitement permet justement à l'instance de réception, non plus simplement d'actualiser son imagination ou les images à partir d'un discours préexistant, mais de vivre avec ses images. Autrement dit, au-delà des impacts émotifs et suggestions extralinguistiques proposés par Umberto Eco, "la conscience affective ${ }^{3}$ " du destinataire est provoquée, stimulée par l'environnement, une atmosphère révélatrice d'une espèce d'épistémè intrinsèque à l'expérience de l'événement.

Le temps-espace ainsi donné à voir devient donc personnifié, humanisé. Mais pour atteindre cette personnification, il faut que la connaissance "du propre" de celui qui le contemple soit conciliable avec l'environnement de l'événement. À cet égard, on ne croira pas que cette harmonie corresponde pour nous à une reproduction respectueuse de l'événement. Autrement dit, nous n'affirmons pas ici que le propre de l'événement est similaire à une mimesis de la nature ou de la réalité, ce qui nous conduirait vers la poétique. Néanmoins, il faudra admettre que le "propre» s'expose dans la chose elle-même, elle est sa nature intrinsèque.

A. Hampaté Bâ (1971, p. 26) déclarait qu'en Afrique, au côté du visible des choses correspond toujours l'invisible. Ainsi, à chaque science apparente correspondra toujours une science 
beaucoup plus profonde "[...] basée sur la conception fondamentale de l'unité de la vie et de l'interrelation, au sein de cette unité». Le propre de cette écriture que nous retrouvons dans la séquence du film d'Anne-Laure Folly ne fait donc pas une reproduction mimétique de la réalité ou de la nature. Elle expose au contraire cette réalité à travers l'unité intrinsèque que l'événement donne à voir, et cela, grâce à la technique cinématographique. Par ailleurs, mettant l'accent sur l'événement, l'expérience, elle fait l'impasse sur la prédominance d'un langage instrumental cinématographique. Nous sommes donc effectivement en face d'une illustration forte de la réflexion heideggerienne sur la technique. Ici, le potentiel de "présentification", un "faire venir », dévoile des capacités non exposées de l'instrument. La révélation du non-instrumental devient à cet effet visible au sein d'une activité interrelationnelle de la même unité, la technique cinématographique. Et c'est aussi à ce niveau que l'écriture cinématographique peut s'illustrer, se révéler, avec des modes opératoires "tactiques". Ceux-ci, offrant à la lecture des caractéristiques personnifiées du temps-espace de l'expérience, demandent aussi au regardant non plus seulement d'activer son encyclopédie, de "comprendre» les images, mais de "vivre» avec ces images, de les "voir", de les vivre dans un acte de contemplation.

C'est donc cette victoire du lieu et des images sur le temps discursif, une victoire faite de "détournements» des objets du discours dominant, une manière de "faire la perruque ${ }^{4}$ " sur les codes scripturaires du langage cinématographiques par des cinéastes africains, que nous assimilons à la "tactique» de Michel de Certeau (1990, p. XLVI):

La "tactique" [est] un calcul qui ne peut pas compter sur son propre, ni donc une frontière qui distingue l'autre comme une totalité visible. La tactique n'a pour lieu que celui de l'autre. Elle s'y insinue, fragmentairement, sans le saisir en entier, sans pouvoir le tenir à distance. Elle ne dispose pas de base pour capitaliser ses avantages, préparer ses expansions et assurer ses indépendances par rapport aux circonstances. Le "propre» est une victoire du lieu sur le temps. Au contraire, du fait de son non-lieu, la tactique dépend du temps, vigilante à y saisir au vol, des possibilités de profit. 
Nous avons montré comment la "stratégie " de l'écriture cinématographique dominante est bâtie sur une relation rationnelle du langage, qui est en soi une des caractéristiques fondamentales de la représentation communicationnelle et qui permet de gommer l'expérience au profit des codes discursifs institutionnalisés. Maintenant, après l'analyse de cette séquence du film d'AnneLaure Folly, nous pouvons nous rendre compte que la «tactique " ne cherche justement pas à "capitaliser ses avantages" comme un sujer de pouvoir le ferait dans le contexte de la représentation. Au contraire, elle s'insinue, fragmentaire, à l'intérieur même des systèmes de la communication stratégique pour tracer son itinéraire. Ainsi, cette tactique scripturaire des cinémas d'Afrique noire procède par «déterritorialisation » et « reterritorialisation", pour reprendre les termes de Deleuze. C'est par infiltration qu'elle arrive à reterritorialiser son espace, son "propre intrinsèque ", et ce, à travers son propre langage. C'est aussi dans cette déterritorialisation et reterritorialisation que nous observons une réception hautement active, similaire à celle des écrivains de la Négritude. Ici aussi, c'est par une rappropriation du langage et de la technique des Occidentaux que les cinéastes africains parviennent à subvertir les codes de la représentation dominante. Ainsi, la technique cinématographique qui servait au départ à montrer une image déformée de l'Afrique est rappropriée par un récepteur africain qui est lui-même devenu un "agent productant".

Enfin, durant cette séquence du Gardien des forces qui illustre une écriture "contemplative», le destinataire africain a tout le temps pour actualiser des références intrinsèques aux images et à l'expérience de l'événement, en l'occurrence, ici, celle d'une tradition orale dont la consultation des voies mystiques est l'enjeu. Mais pour une instance de réception dont les modes de lecture seront exclusivement ceux de la représentation dominante - à partir du moment où son encyclopédie ne contient pas une affectivité en rapport avec les images, mais plutôt des codes linguistiques ancrés dans une stratégie scripturaire (qui se trouve ici dynamitée) - , la vie qu'il donnera à ces images restera limitée au sens que le sujet producteur individuel aura imposé. 
Conclusion : limites des applications du sujet dominant

Nous insistons sur le fait que c'est dans une affectivité intrinsèque à une épistémè collective qu'il faut chercher les modes opératoires des articulations scripturaires dans les cinémas d'Afrique noire. Nous soulignons aussi que ces modes opératoires écartent en soi la possibilité d'un sujet individuel dont les pouvoirs transcenderaient l'expérience de l'événement.

Le développement historique des cinémas d'Afrique noire montrait qu'il fallait faire front commun pour articuler une politique de création. La partie de la charte de la FEPACI qui bannit tout créateur individuel est un bon exemple de cet engagement. Mais déjà, cette pensée tire sa graine dans un ordre social impliquant la mort de tout individualisme, celui d'un sujet producteur comme celui que l'on décèle dans le contenu d'une création (par le gros plan, le narrateur omniscient, etc.).

Revenons une dernière fois sur le gros plan puisqu'il est pour beaucoup dans les conclusions que nous avons tirées au cours de la présente étude. Dans les cinémas dominants, les gros plans légitiment généralement le côté psychologique d'un personnage. Comme le dit Marcel Martin (1985, p. 43), "le gros plan [...] correspond à un envahissement du champ de la conscience, à une tension mentale considérable, à un mode de pensée obsessionnel. [...] Le gros plan suggère alors une forte tension mentale chez le personnage [...]". Et il demande au destinataire de pénétrer dans le for intérieur du personnage. Ainsi fonctionne L'Enfant noir de Laurent Chevalier. D'autre part, notre description d'une séquence du Gardien des forces a permis de constater que tous les gros plans sont axés non sur les personnes mais sur des fétiches. Le marabout, protagoniste de la scène, ne reçoit aucun traitement privilégiant sa position dans le cadrage. Même dans la perspective d'une analyse esthétique du cadrage, les fétiches sont toujours au centre de l'image (même quand d'autres personnages font partie du même cadrage), ce qui accentue leur place par rapport à ce qui est raconté. Par ailleurs, au moment où ils sont seuls dans le cadrage, ils occupent tout le champ et deviennent automatiquement, encore une fois, l'objet d'analyse. En d'autres termes, au lieu de chercher la psychologie du marabout ou des personnages impliqués dans la cérémonie, les fétiches deviennent ce qu'il faut absolument "voir ". 
C'est dire que, dans le film d'Anne-Laure Folly, le psychologisme du gros plan n'est d'aucune utilité. Le film le déclare non avenu. Ce n'est donc pas à partir du gros plan que l'on pourra analyser les pulsions névrotiques, les champs de conscience et les pensées obsessionnelles de la collectivité africaine. Car précisément, il s'agit d'échapper aux discours dominants que manifeste une certaine écriture cinématographique basée sur la primauté du sujet et la nécessité psychologique d'analyser ce sujet. Perverse et polymorphe, dit Deleuze, la psychanalyse "casse toutes les productions du désir, elle écrase toutes les formations d'énoncés. Par-là, elle brise l'agencement sur ses deux faces, l'agencement machinique de désir, l'agencement collectif d'énonciation" (Deleuze et Parnet, 1996, p. 95). Et ce dernier constat est particulièrement important pour une analyse de l'écriture dans les cinémas d'Afrique noire puisque à son sujet on parle souvent d'énoncés issus du collectif.

L'examen de l'écriture des cinémas d'Afrique noire doit donc aujourd'hui interroger les théories dominantes et leurs présupposés universalistes. En effet, il apparait évident qu'on ne peut plus imposer les codes d'un langage à des films qui n'ont rien à faire avec ces codes. Les défis futurs? En plus d'une critique historique impliquant une relecture des modes opératoires propres, essayer de faire une lecture intrinsèque en profondeur des conditions et possibilités que les films proposent. C'est cela, l'étude avec l'agencement: un espace de réflexion à travers lequel une constellation d'éléments émerge. Favoriser l'un aux dépens de l'autre contribue à la perversité d'un discours unique.

Concordia University

\section{NOTES}

1. Les cinémas d'Afrique se retrouvent donc aujourd'hui encore dans une position similaire à celle du cinéma français sous l'Occupation, alors que les artistes comme Jacques Prévert et Marcel Carné arrivaient à déjouer la censure allemande et s'imposaient avec une écriture remarquablement riche. Nous pensons entre autres aux Visiteurs du soir (1942), à Quai des brumes (1938), aux Enfants du paradis (1945), réalisés sous la censure allemande tout en dénonçant les maux de l'Occupation.

2. Selon la poétique artistotélicienne et les applications modernes de son principe de l'"étendue " dans les cinémas dominants (c'est-à-dire la vitesse d'action du 
montage, la caractérisation psychologique des personnages que les gros plans accentuent, etc.).

3. Nous empruntons ce concept d'affectivité à Sartre (L'Imaginaire, Paris: Gallimard, 1986). Et par "affectivité" nous entendons une activité similaire mais beaucoup plus large que l'"encyclopédie" d'Umberto Eco (1985). Pour être un peu plus complète, l'affectivité serait alors une combinatoire avec le concept d'épistémè de Foucault (Les Mots et les choses, Paris: Gallimard, 1966) qui permettrait néanmoins la possibilité d'intégration de codes linguistiques et extra-linguistiques qu'on retrouve dans l'encyclopédie qui, en soi, implique aussi une actualisation de l'écriture dans sa lecture. La combinatoire encyclopédielépistémè nous permet en effet de pousser cette déclaration un peu plus loin, car elle reconnaît d'une part les codes sémiologiques - légitimant ainsi la présence d'un langage - et d'autre part la présence de liens phénoménologiques préexistants dans une activité engageant un média, quel qu'il soit.

4. Il s'agit d'une vieille expression française évoquant cette possibilité qu'avaient les employés à voler quelques petits biens de leurs patrons, cela malgré les interdits et tous les systèmes mis en place pour contrecarrer ces vols.

\section{OUVRAGES CITÉS}

Anonyme. "Le Cinématographe à la conquête du monde ". Ciné-Journal, n" 36 (2328 avril 1909).

Bâ, A. Hampaté. Aspect de la civilisation africaine. Paris: Présence africaine, 1971.

Barlet, Olivier. Les Cinémas d'Afrique noire. Le regard en question. Paris : L'Harmattan, 1996.

Convents, Guido. Préhistoire du cinéma en Afrique. A la recherche des images oubliées. Bruxelles: OCIC, 1989.

De Certeau, Michel. L'Invention du quotidien. L'art de faire. Paris: Gallimard, 1990.

Debrix, Jean-René. "Documents d'Afrique noire et de Madagascar". Afrique-Action: Afrique contemporaine, $\mathrm{n}^{\infty}$ 38-39, 7 ème année, p. 7.

Deleuze, Gilles et Claire Parnet. Dialogues. Paris: Champs/Flammarion, 1996.

Eco, Umberto. Lector in Fabula. Paris : Grasset, 1985.

Foucault, Michel. Les Mots et les choses. Paris : Gallimard, 1966.

Hall, Stuart. "Encoding/Decoding", Culture, Media, Language. London: Hall et al., 1980.

Heidegger, Martin. The Question Concerning Technology of Other Essays. New York: Harper \& Row, 1977 (1954).

Hodeir, Catherine et Michel Pierre. 1931 : L'exposition coloniale. Bruxelles: Éditions Complexe, 1991.

J. C., "La maladie du sommeil ", Pathé Journal, n 9 (décembre 1912).

Kesteloot, Lilian. Négriture et situation coloniale. Yaoundé: Éditions Clé, 1968.

Martin, Marcel. Le Langage cinématographique. Paris: Cerf, 1985.

M. G. "Le noir est à la mode». Cinémonde, $n^{\circ} 36$ (juin 1929).

Ravault, René-Jean. "Colonialisme culturel et "coerséductions" autochotones". Communication, vol. 8, $\mathrm{n}^{\circ} 1,1986$, p. 55-101.

Saï, Edward. Orientalism. New York: Vintage, 1978.

Sartre, Jean-Paul. L'Imaginaire. Paris: Gallimard, 1986. 